\title{
Episodic Learning with Control Lyapunov Functions for Uncertain Robotic Systems*
}

\author{
Andrew J. Taylor ${ }^{1}$, Victor D. Dorobantu ${ }^{1}$, Hoang M. Le, Yisong Yue, Aaron D. Ames
}

\begin{abstract}
Many modern nonlinear control methods aim to endow systems with guaranteed properties, such as stability or safety, and have been successfully applied to the domain of robotics. However, model uncertainty remains a persistent challenge, weakening theoretical guarantees and causing implementation failures on physical systems. This paper develops a machine learning framework centered around Control Lyapunov Functions (CLFs) to adapt to parametric uncertainty and unmodeled dynamics in general robotic systems. Our proposed method proceeds by iteratively updating estimates of Lyapunov function derivatives and improving controllers, ultimately yielding a stabilizing quadratic program modelbased controller. We validate our approach on a planar Segway simulation, demonstrating substantial performance improvements by iteratively refining on a base model-free controller.
\end{abstract}

\section{INTRODUCTION}

The use of Control Lyapunov Functions (CLFs) [5], [38] for nonlinear control of robotic systems is becoming increasingly popular [26], [17], [29], often utilizing quadratic program controllers [3], [2], [17]. While effective, one major challenge is the need for extensive tuning, which is largely due to modeling deficiencies such as parametric error and unmodeled dynamics (cf. [26]). While there has been much research in developing robust control methods that maintain stability under uncertainty (e.g., via input-to-state stability [39]) or in adapting to limited forms of uncertainty (e.g., adaptive control [23],[20]), relatively little work has been done on systematically reducing uncertainty while maintaining stability for general function classes of models.

We take a machine learning approach to address the above limitations. Learning-based approaches have already shown great promise for controlling imperfectly modeled robotic platforms [22], [35]. Successful learning-based approaches have typically focused on learning model-based uncertainty [6], [9], [8], [37], or direct model-free controller design [25], [36], [14], [42], [24].

We are particularly interested in learning-based approaches that guarantee Lyapunov stability [21]. From that perspective, the bulk of previous work has focused on using learning to construct a Lyapunov function [31], [12], [30], or to assess the region of attraction for a Lyapunov function [10], [7].

*This work was supported by Google Brain Robotics and DARPA Award HR00111890035

${ }^{1}$ Both authors contributed equally.

All authors are with the Department of Computing and Mathematical Sciences, California Institute of Technology, Pasadena, CA 91125, USA ajtaylordcaltech.edu, vdoroban@caltech.edu, hmle@caltech.edu, yyuedcaltech.edu, amesecaltech.edu

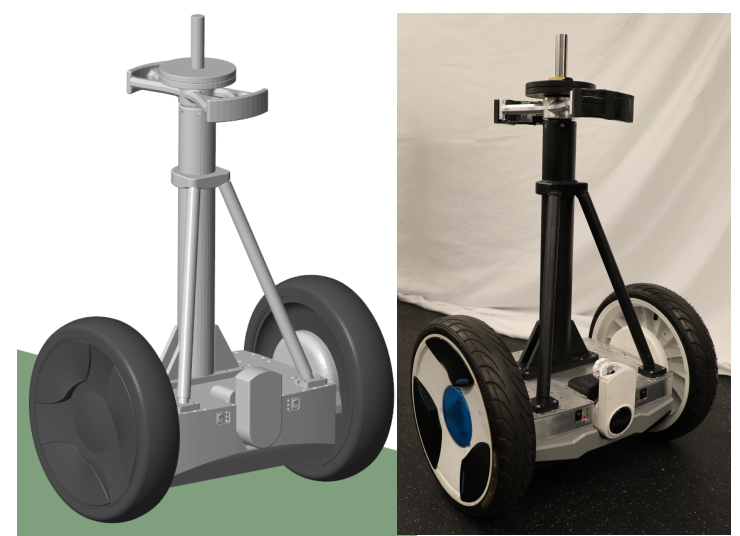

Fig. 1. CAD model \& physical system, a modified Ninebot Segway.

One limitation of previous work is the learning is conducted over the full-dimensional state space, which can be data inefficient. We instead constructively prescribe a CLF, and focus on learning only the necessary information to choose control inputs that achieve the associated stability guarantees, which can be much lower-dimensional.

One challenge in developing learning-based methods for controller improvement is how best to collect training data that accurately reflects the desired operating environment and control goals. In particular, exhaustive data collection typically scales exponentially with dimensionality of the joint state and control output space, and so should be avoided. But first pre-collecting data upfront can lead to poor performance as downstream control behavior may enter states that are not present in the pre-collected training data. We will leverage episodic learning approaches such as Dataset Aggregation (DAgger) [33] to address these challenges in a data-efficient manner, and lead to iteratively refined controllers.

In this paper we present a novel episodic learning approach that utilizes CLFs to iteratively improve controller design while maintaining stability. To the best of our knowledge, our approach is the first that integrates CLFs and general supervised learning (e.g., including deep learning) in a mathematically integrated way. Another distinctive aspect is that our approach performs learning on the projection of state dynamics onto the CLF time derivative, which can be much lower dimensional than learning the full state dynamics or the region of attraction.

Our paper is organized as follows. Section II presents a review of input-output feedback linearization with a focus on constructing CLFs for unconstrained robotic systems. Section III discusses model uncertainty of a general robotic 
system and establishes assumptions on the structure of this uncertainty. These assumptions allow us to prescribe a CLF for the true system, but leave open the question of how to model its time derivative. Section IV provides an episodic learning approach to iteratively improving a model of the time derivative of the CLF. We also present a variant of optimal CLF-based control that integrates the learned representation. Finally, Section $\mathrm{V}$ provides simulation results on a model of a modified Ninebot by Segway $E+$, seen in Fig. 1. We also provide a Python software package (LyaPy) implementing our experiments and learning framework 1

\section{PRELIMINARIES ON CLFS}

This section provides a brief review of input-output feedback linearization, a control technique which can be used to synthesize a CLF. The resulting CLF will be used to quantify the impact of model uncertainty and specify the learning problem outlined in Section III.

\section{A. Input-Output Linearization}

Input-Output Linearization is a nonlinear control method that creates stable linear dynamics for a selected set of outputs of a system [21]. The relevance of Input-Output Linearization is that it provides a constructive way to generate Lyapunov functions for the class of affine robotic control systems. Consider a configuration space $\mathcal{Q} \subseteq \mathbb{R}^{n}$ and an input space $\mathcal{U} \subseteq \mathbb{R}^{m}$. Assume $\mathcal{Q}$ is path-connected and nonempty. Consider a control system specified by:

$$
\mathbf{D}(\mathbf{q}) \ddot{\mathbf{q}}+\underbrace{\mathbf{C}(\mathbf{q}, \dot{\mathbf{q}}) \dot{\mathbf{q}}+\mathbf{G}(\mathbf{q})}_{\mathbf{H}(\mathbf{q}, \dot{\mathbf{q}})}=\mathbf{B u},
$$

with generalized coordinates $\mathbf{q} \in \mathcal{Q}$, coordinate rates $\dot{\mathbf{q}} \in$ $\mathbb{R}^{n}$, input $\mathbf{u} \in \mathcal{U}$, inertia matrix $\mathbf{D}: \mathcal{Q} \rightarrow \mathbb{S}_{++}^{n}$, centrifugal and Coriolis terms $\mathbf{C}: \mathcal{Q} \times \mathbb{R}^{n} \rightarrow \mathbb{R}^{n \times n}$, gravitational forces $\mathbf{G}: \mathcal{Q} \rightarrow \mathbb{R}^{n}$, and static actuation matrix $\mathbf{B} \in \mathbb{R}^{n \times m}$. Here $\mathbb{S}_{++}^{n}$ denotes the set of $n \times n$ symmetric positive definite matrices. Define twice-differentiable outputs y : $\mathcal{Q} \rightarrow \mathbb{R}^{k}$, with $k \leq m$, and assume each output has relative degree 2 on some domain $\mathcal{R} \subseteq \mathcal{Q}$ (see [34] for more details). Consider the time interval $\mathcal{I}=\left[t_{0}, t_{f}\right]$ for initial and final times $t_{0}, t_{f}$ satisfying $t_{f}>t_{0}$ and define twice-differentiable time-dependent desired outputs $\mathbf{y}_{d}: \mathcal{I} \rightarrow \mathbb{R}^{k}$ with $\mathbf{r}(t)=$ $\left[\begin{array}{ll}\mathbf{y}_{d}(t)^{\top} & \dot{\mathbf{y}}_{d}(t)^{\top}\end{array}\right]^{\top}$. The error between the outputs and the desired outputs (commonly referred to as virtual constraints [44]) yields the dynamic system:

$$
\begin{aligned}
\frac{\mathrm{d}}{\mathrm{d} t}\left[\begin{array}{c}
\mathbf{y}(\mathbf{q})-\mathbf{y}_{d}(t) \\
\dot{\mathbf{y}}(\mathbf{q}, \dot{\mathbf{q}})-\dot{\mathbf{y}}_{d}(t)
\end{array}\right]= & \overbrace{\left[\begin{array}{c}
\frac{\partial \dot{\mathbf{y}}}{\partial \mathbf{q}} \dot{\mathbf{q}}-\frac{\partial \mathbf{y}}{\partial \mathbf{q}} \mathbf{D}(\mathbf{q})^{-1} \mathbf{H}(\mathbf{q}, \dot{\mathbf{q}})
\end{array}\right]}^{\left.\frac{\partial \mathbf{y}}{\dot{\mathbf{q}}} \dot{\mathbf{q}}\right)} \\
& -\underbrace{\left[\begin{array}{c}
\dot{\mathbf{y}}_{d}(t) \\
\ddot{\mathbf{y}}_{d}(t)
\end{array}\right]}_{\dot{\dot{\mathbf{r}}}(t)}+\underbrace{\left[\begin{array}{c}
\mathbf{0}_{k \times m} \\
\frac{\partial \mathbf{y}}{\partial \mathbf{q}} \mathbf{D}(\mathbf{q})^{-1} \mathbf{B}
\end{array}\right]}_{\mathbf{g}(\mathbf{q})} \mathbf{u},
\end{aligned}
$$

noting that $\frac{\partial \dot{\mathbf{y}}}{\partial \dot{\mathbf{q}}}=\frac{\partial \mathbf{y}}{\partial \mathbf{q}}$. For all $\mathbf{q} \in \mathcal{R}, \mathbf{g}(\mathbf{q})$ is full rank by the relative degree assumption. Define $\boldsymbol{\eta}: \mathcal{Q} \times \mathbb{R}^{n} \times \mathcal{I} \rightarrow \mathbb{R}^{2 k}$, $\widetilde{\mathbf{f}}: \mathcal{Q} \times \mathbb{R}^{n} \rightarrow \mathbb{R}^{k}$, and $\widetilde{\mathbf{g}}: \mathcal{Q} \rightarrow \mathbb{R}^{k \times m}$ as:

$$
\begin{aligned}
\boldsymbol{\eta}(\mathbf{q}, \dot{\mathbf{q}}, t) & =\left[\begin{array}{c}
\mathbf{y}(\mathbf{q})-\mathbf{y}_{d}(t) \\
\dot{\mathbf{y}}(\mathbf{q}, \dot{\mathbf{q}})-\dot{\mathbf{y}}_{d}(t)
\end{array}\right] \\
\widetilde{\mathbf{f}}(\mathbf{q}, \dot{\mathbf{q}}) & =\frac{\partial \dot{\mathbf{y}}}{\partial \mathbf{q}} \dot{\mathbf{q}}-\frac{\partial \mathbf{y}}{\partial \mathbf{q}} \mathbf{D}(\mathbf{q})^{-1} \mathbf{H}(\mathbf{q}, \dot{\mathbf{q}}) \\
\widetilde{\mathbf{g}}(\mathbf{q}) & =\frac{\partial \mathbf{y}}{\partial \mathbf{q}} \mathbf{D}(\mathbf{q})^{-1} \mathbf{B},
\end{aligned}
$$

and assume $\mathcal{U}=\mathbb{R}^{m}$. The input-output linearizing control input is specified by:

$$
\mathbf{u}(\mathbf{q}, \dot{\mathbf{q}}, t)=\widetilde{\mathbf{g}}(\mathbf{q})^{\dagger}\left(-\widetilde{\mathbf{f}}(\mathbf{q}, \dot{\mathbf{q}})+\ddot{\mathbf{y}}_{d}(t)+\boldsymbol{\nu}(\mathbf{q}, \dot{\mathbf{q}}, t)\right),
$$

with auxiliary input $\boldsymbol{\nu}(\mathbf{q}, \dot{\mathbf{q}}, t) \in \mathbb{R}^{k}$ for all $\mathbf{q} \in \mathcal{Q}$, $\dot{\mathbf{q}} \in \mathbb{R}^{n}$, and $t \in \mathcal{I}$, where $\dagger$ denotes the Moore-Penrose pseudoinverse. This controller used in 22 generates linear output dynamics of the form:

$$
\dot{\boldsymbol{\eta}}(\mathbf{q}, \dot{\mathbf{q}}, t)=\underbrace{\left[\begin{array}{ll}
\mathbf{0}_{k \times k} & \mathbf{I}_{k \times k} \\
\mathbf{0}_{k \times k} & \mathbf{0}_{k \times k}
\end{array}\right]}_{\mathbf{F}} \boldsymbol{\eta}(\mathbf{q}, \dot{\mathbf{q}}, t)+\underbrace{\left[\begin{array}{c}
\mathbf{0}_{k \times k} \\
\mathbf{I}_{k \times k}
\end{array}\right]}_{\mathbf{G}} \boldsymbol{\nu}(\mathbf{q}, \dot{\mathbf{q}}, t),
$$

where $(\mathbf{F}, \mathbf{G})$ are a controllable pair. Defining $\mathbf{K}=$ $\left[\begin{array}{ll}\mathbf{K}_{p} & \mathbf{K}_{d}\end{array}\right]$ where $\mathbf{K}_{p}, \mathbf{K}_{d} \in \mathbb{S}_{++}^{k}$, the auxiliary control input $\boldsymbol{\nu}(\mathbf{q}, \dot{\mathbf{q}}, t)=-\mathbf{K} \boldsymbol{\eta}(\mathbf{q}, \dot{\mathbf{q}}, t)$ induces output dynamics:

$$
\dot{\boldsymbol{\eta}}(\mathbf{q}, \dot{\mathbf{q}}, t)=\mathbf{A}_{c l} \boldsymbol{\eta}(\mathbf{q}, \dot{\mathbf{q}}, t)
$$

where $\mathbf{A}_{c l}=\mathbf{F}-\mathbf{G K}$ is Hurwitz. This implies the desired output trajectory $\mathbf{y}_{d}$ is exponentially stable. This conclusion allows us to construct a Lyapunov function for the system using converse theorems found in [21]. With $\mathbf{A}_{c l}$ Hurwitz, for any $\mathbf{Q} \in \mathbb{S}_{++}^{2 k}$, there exists a unique $\mathbf{P} \in \mathbb{S}_{++}^{2 k}$ such that the Continuous Time Lyapunov Equation (CTLE):

$$
\mathbf{A}_{c l}^{\top} \mathbf{P}+\mathbf{P A}_{c l}=-\mathbf{Q}
$$

is satisfied. Let $\mathcal{C}=\left\{\boldsymbol{\eta}(\mathbf{q}, \dot{\mathbf{q}}, t):(\mathbf{q}, \dot{\mathbf{q}}) \in \mathcal{R} \times \mathbb{R}^{n}, t \in \mathcal{I}\right\}$. Then $V(\boldsymbol{\eta})=\boldsymbol{\eta}^{\top} \mathbf{P} \boldsymbol{\eta}$, implicitly a function of $\mathbf{q}$, $\dot{\mathbf{q}}$, and $t$, is a Lyapunov function certifying exponential stability of 8 on $\mathcal{C}$ satisfying:

$$
\begin{array}{r}
\lambda_{\min }(\mathbf{P})\|\boldsymbol{\eta}\|_{2}^{2} \leq V(\boldsymbol{\eta}) \leq \lambda_{\max }(\mathbf{P})\|\boldsymbol{\eta}\|_{2}^{2} \\
\dot{V}(\boldsymbol{\eta}) \leq-\lambda_{\min }(\mathbf{Q})\|\boldsymbol{\eta}\|_{2}^{2},
\end{array}
$$

for all $\boldsymbol{\eta} \in \mathcal{C}$. Here $\lambda_{\min }(\cdot)$ and $\lambda_{\max }(\cdot)$ denote the minimum and maximum eigenvalues of a symmetric matrix, respectively. Alternatively, a Lyapunov function of the same form can be constructed directly from (7) using the Continuous Algebraic Riccati Equation (CARE) [21].

\section{B. Control Lyapunov Functions}

The preceding formulation of a Lyapunov function required the choice of the specific control law given in (6). For optimality purposes, it may be desirable to choose a different control input for the system, thus motivating the following definition. Let $\mathcal{C} \subseteq \mathbb{R}^{2 k}$. A function $V: \mathbb{R}^{2 k} \rightarrow \mathbb{R}_{+}$is a Control Lyapunov Function (CLF) for (1) on $\mathcal{C}$ certifying 
exponential stability if there exist constants $c_{1}, c_{2}, c_{3}>0$ such that:

$$
\begin{gathered}
c_{1}\|\boldsymbol{\eta}\|_{2}^{2} \leq V(\boldsymbol{\eta}) \leq c_{2}\|\boldsymbol{\eta}\|_{2}^{2} \\
\inf _{\mathbf{u} \in \mathcal{U}} \dot{V}(\boldsymbol{\eta}, \mathbf{u}) \leq-c_{3}\|\boldsymbol{\eta}\|_{2}^{2},
\end{gathered}
$$

for all $\boldsymbol{\eta} \in \mathcal{C}$. We see that the previously constructed Lyapunov function satisfying (10) satisfies (11) by choosing the control input specified in (6). In the absence of a specific control input, we may write the Lyapunov function time derivative as:

$$
\dot{V}(\boldsymbol{\eta}, \mathbf{u})=\frac{\partial V}{\partial \boldsymbol{\eta}} \dot{\boldsymbol{\eta}}=\frac{\partial V}{\partial \boldsymbol{\eta}}(\mathbf{f}(\mathbf{q}, \dot{\mathbf{q}})-\dot{\mathbf{r}}(t)+\mathbf{g}(\mathbf{q}) \mathbf{u}) .
$$

Information about the dynamics is encoded within the scalar function $\dot{V}$, offering a reduction in dimensionality which will become relevant later in learning. Also note that $\dot{V}$ is affine in $\mathbf{u}$. This leads to the class of quadratic program based controllers given by:

$$
\begin{aligned}
\mathbf{u}(\mathbf{q}, \dot{\mathbf{q}}, t)=\underset{\mathbf{u} \in \mathcal{U}}{\arg \min } & \frac{1}{2} \mathbf{u}^{\top} \mathbf{M} \mathbf{u}+\mathbf{s}^{\top} \mathbf{u}+r \\
\text { s.t. } & \dot{V}(\boldsymbol{\eta}, \mathbf{u}) \leq-c_{3}\|\boldsymbol{\eta}\|_{2}^{2},
\end{aligned}
$$

for $\mathbf{M} \in \mathbb{S}_{+}^{m}, \mathbf{s} \in \mathbb{R}^{m}$, and $r \in \mathbb{R}$, provided $\mathcal{U}$ is a polyhedron. Here $\mathbb{S}_{+}^{m}$ denotes the set of $m \times m$ symmetric positive semi-definite matrices.

\section{UNCERTAINTY MODELS \& LEARNING}

This section defines the class of model uncertainty we consider in this work and investigates its impact on the control system, and concludes with motivation for a datadriven approach to mitigate this impact.

\section{A. Uncertainty Modeling Assumptions}

As defined in Section III, we consider affine robotic control systems that evolve under dynamics described by (1). In practice, we do not know the dynamics of the system exactly, and instead develop our control systems using the estimated model:

$$
\widehat{\mathbf{D}}(\mathbf{q}) \ddot{\mathbf{q}}+\underbrace{\widehat{\mathbf{C}}(\mathbf{q}, \dot{\mathbf{q}}) \dot{\mathbf{q}}+\widehat{\mathbf{G}}(\mathbf{q})}_{\widehat{\mathbf{H}}(\mathbf{q}, \dot{\mathbf{q}})}=\widehat{\mathbf{B}} \mathbf{u} .
$$

We assume the estimated model (14) satisfies the relative degree condition on the domain $\mathcal{R}$, and thus may use the method of feedback linearization to produce a Control Lyapunov Function (CLF), $V$, for the system. Using the definitions established in 2) in conjunction with the estimated model, we see that true system evolves as:

$$
\begin{aligned}
\dot{\eta}= & \widehat{\mathbf{f}}(\mathbf{q}, \dot{\mathbf{q}})-\dot{\mathbf{r}}(t)+\widehat{\mathbf{g}}(\mathbf{q}) \mathbf{u} \\
& +\underbrace{(\underbrace{}_{\mathbf{g}(\mathbf{q})-\widehat{\mathbf{g}}(\mathbf{q})})}_{\mathbf{A}(\mathbf{q})} \mathbf{u}+\underbrace{\mathbf{f}(\mathbf{q}, \dot{\mathbf{q}})-\widehat{\mathbf{f}}(\mathbf{q}, \dot{\mathbf{q}})}_{\mathbf{b}(\mathbf{q}, \dot{\mathbf{q}})} .
\end{aligned}
$$

We note the following features of modeling uncertainty in this fashion:

- Uncertainty is allowed to enter the system dynamics via parametric error as well as through completely unmodeled dynamics. In particular, the function $\mathbf{H}$ can capture a wide variety of nonlinear behavior and only needs to be Lipschitz continuous.

- This formulation explicitly allows uncertainty in how the input is introduced into the dynamics via uncertainty in the inertia matrix $\mathbf{D}$ and static actuation matrix $\mathbf{B}$. This definition of uncertainty is also compatible with a dynamic actuation matrix B : $\mathcal{Q} \times \mathbb{R}^{n} \rightarrow \mathbb{R}^{n \times m}$ given proper assumptions on the relative degree of the system.

Given this formulation of our uncertainty, we make the following assumptions of the true dynamics:

Assumption 1. The true system is assumed to be deterministic, time invariant, and affine in the control input.

Assumption 2. The CLF $V$, formulated for the estimated model, is a CLF for the true system.

It is sufficient to assume that the true system have relative degree 2 on the domain $\mathcal{R}$ to satisfy Assumption 2 This holds since the true values of $\widetilde{\mathbf{f}}$ and $\widetilde{\mathbf{g}}$, if known, enable choosing control inputs as in (6) that respect the same linear output dynamics (8). Given that $V$ is a CLF for the true system, its time derivative under uncertainty is given by:

$$
\begin{aligned}
\dot{V}(\boldsymbol{\eta}, \mathbf{u})= & \overbrace{\frac{\partial V}{\partial \boldsymbol{\eta}}(\widehat{\mathbf{f}}(\mathbf{q}, \dot{\mathbf{q}})-\dot{\hat{r}}(\boldsymbol{\eta}, \mathbf{u})}^{\hat{\mathbf{r}}(t)+\widehat{\mathbf{g}}(\mathbf{q}) \mathbf{u})} \\
& +\underbrace{\frac{\partial V}{\partial \boldsymbol{\eta}} \mathbf{A}(\mathbf{q})}_{\mathbf{a}(\boldsymbol{\eta}, \mathbf{q})^{\top}} \mathbf{u}+\underbrace{\frac{\partial V}{\partial \boldsymbol{\eta}} \mathbf{b}(\mathbf{q}, \dot{\mathbf{q}})}_{b(\boldsymbol{\eta}, \mathbf{q}, \dot{\mathbf{q}})},
\end{aligned}
$$

for all $\boldsymbol{\eta} \in \mathbb{R}^{2 k}$ and $\mathbf{u} \in \mathcal{U}$. While $V$ is a CLF for the true system, it is no longer possible to determine if a specific control value will satisfy the derivative condition in (11) due to the unknown components a and $b$. Rather than form a new Lyapunov function, we seek to better estimate the Lyapunov function derivative $\dot{V}$ to enable control selection that satisfies the exponential stability requirement. This estimate should be affine in the control input, enabling its use in the controller described in 13. Instead of learning the unknown dynamics terms $\mathbf{A}$ and $\mathbf{b}$, which scale with both the dimension of the configuration space and the number of inputs, we will learn the terms a and $b$, which scale only with the number of inputs. In the case of the planar Segway model we simulate, we reduce the number of learned components from 4 to 2 (assuming kinematics are known). These learned representations need to accurately capture the uncertainty over the domain in which the system is desired to evolve to ensure stability during operation.

\section{B. Motivating a Data-Driven Learning Approach}

The formulation from 15 and 16 defines a general class of dynamics uncertainty. It is natural to consider a datadriven method to estimate the unknown quantities a and $b$ over the domain of the system. To motivate our learningbased framework, first consider a simple approach of learning a and $b$ via supervised regression [19]: we operate the system 
using some given state-feedback controller to gather data points along the system's evolution and learn a function that approximates a and $b$ via supervised learning.

Concretely, let $\mathbf{q}_{0} \in \mathcal{Q}$ be an initial configuration. An experiment is defined as the evolution of the system over a finite time interval from the initial condition $\left(\mathbf{q}_{0}, \mathbf{0}\right)$ using a discrete-time implementation of the given controller. A resulting discrete-time state history is obtained, which is then transformed with Lyapunov function $V$ and finally differentiated numerically to estimate $\dot{V}$ throughout the experiment. This yields a data set comprised of input-output pairs:

$$
D=\left\{\left(\left(\mathbf{q}_{i}, \dot{\mathbf{q}}_{i}, \boldsymbol{\eta}_{i}, \mathbf{u}_{i}\right), \dot{V}_{i}\right)\right\}_{i=1}^{N} \subseteq\left(\mathcal{Q} \times \mathbb{R}^{n} \times \mathbb{R}^{2 k} \times \mathcal{U}\right) \times \mathbb{R} .
$$

Consider a class $\mathcal{H}_{\mathbf{a}}$ of nonlinear functions mapping from $\mathbb{R}^{2 k} \times \mathcal{Q}$ to $\mathbb{R}^{m}$ and a class $\mathcal{H}_{b}$ of nonlinear functions mapping from $\mathbb{R}^{2 k} \times \mathcal{Q} \times \mathbb{R}^{n}$ to $\mathbb{R}$. For a given $\widehat{\mathbf{a}} \in \mathcal{H}_{\mathbf{a}}$ and $\widehat{b} \in \mathcal{H}_{b}$, define $\widehat{\dot{W}}$ as:

$$
\widehat{\dot{W}}(\boldsymbol{\eta}, \mathbf{q}, \dot{\mathbf{q}}, \mathbf{u})=\widehat{\dot{V}}(\boldsymbol{\eta}, \mathbf{u})+\widehat{\mathbf{a}}(\boldsymbol{\eta}, \mathbf{q})^{\top} \mathbf{u}+\widehat{b}(\boldsymbol{\eta}, \mathbf{q}, \dot{\mathbf{q}}),
$$

and let $\mathcal{H}$ be the class of all such estimators mapping $\mathbb{R}^{2 k} \times$ $\mathcal{Q} \times \mathbb{R}^{n} \times \mathcal{U}$ to $\mathbb{R}$. Defining a loss function $\mathcal{L}: \mathbb{R} \times \mathbb{R} \rightarrow \mathbb{R}_{+}$, the supervised regression task is then to find a function in $\mathcal{H}$ via empirical risk minimization (ERM):

$$
\inf _{\substack{\hat{a} \in \mathcal{H}_{\mathbf{a}} \\ \widehat{b} \in \mathcal{H}_{b}}} \frac{1}{N} \sum_{i=1}^{N} \mathcal{L}\left(\widehat{\dot{W}}\left(\boldsymbol{\eta}_{i}, \mathbf{q}_{i}, \dot{\mathbf{q}}_{i}, \mathbf{u}_{i}\right), \dot{V}_{i}\right) .
$$

This experiment protocol can be executed either in simulation or directly on hardware. While being simple to implement, supervised learning critically assumes independently and identically distributed (i.i.d) training data. Each experiment violates this assumption, as the regression target of each data point is coupled with the input data of the next time step. As a consequence, standard supervised learning with sequential, non-i.i.d data collection often leads to error cascades [24].

\section{INTEGRATING EPISODIC LEARNING \& CLFS}

In this section we present the main contribution of this work: an episodic learning algorithm that captures the uncertainty present in the Lyapunov function derivative in a learned model and utilizes it in a quadratic program based controller.

\section{A. Episodic Learning Framework}

Episodic learning refers to learning procedures that iteratively alternates between executing an intermediate controller (also known as a roll-out in reinforcement learning [22]), collecting data from that roll-out, and designing a new controller using the newly collected data. Our approach integrates learning $\mathbf{a}$ and $b$ with improving the performance and stability of the control policy $\mathbf{u}$ in such an iterative fashion. First, assume we are given a nominal state-feedback controller $\mathbf{u}: \mathcal{Q} \times \mathbb{R}^{n} \times \mathcal{I} \rightarrow \mathcal{U}$. With an estimator $\widehat{\dot{W}} \in \mathcal{H}$

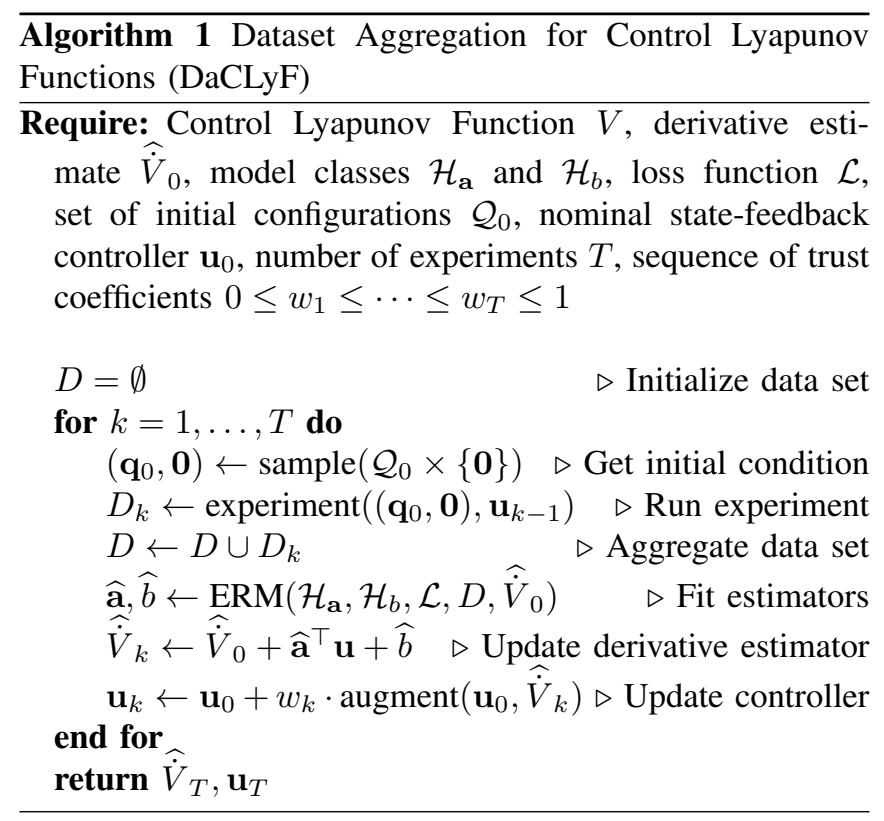

as defined in 18 , we specify an augmenting controller as:

$$
\begin{aligned}
\mathbf{u}^{\prime}(\mathbf{q}, \dot{\mathbf{q}}, t)=\underset{\mathbf{u}^{\prime} \in \mathbb{R}^{m}}{\arg \min } J\left(\mathbf{u}^{\prime}\right) & \\
\text { s.t. } \widehat{\dot{W}} & \left.\boldsymbol{\eta}, \mathbf{q}, \dot{\mathbf{q}}, \mathbf{u}(\mathbf{q}, \dot{\mathbf{q}}, t)+\mathbf{u}^{\prime}\right) \leq-c_{3}\|\boldsymbol{\eta}\|_{2}^{2} \\
& \mathbf{u}(\mathbf{q}, \dot{\mathbf{q}}, t)+\mathbf{u}^{\prime} \in \mathcal{U},
\end{aligned}
$$

where $J: \mathbb{R}^{m} \rightarrow \mathbb{R}$ is any positive semi-definite quadratic cost function.

Our goal is to use this new controller to obtain better estimates of a and $b$. One option, as seen in Section III-B, is to perform experiments and use conventional supervised regression to update $\widehat{a}$ and $\widehat{b}$. To overcome the limitations of conventional supervised learning, we leverage reduction techniques: a sequential prediction problem is reduced to a sequence of supervised learning problems over multiple episodes [15], [32]. In particular, in each episode, an experiment generates data using a different controller. The data set is aggregated and a new ERM problem is solved after each episode. Our episodic learning implementation is inspired by the Data Aggregation algorithm (DAgger) [32], with some key differences:

- DAgger is a reinforcement learning algorithm, which trains a policy directly in each episode using optimal computational oracles. Our algorithm defines a controller indirectly via a CLF to ensure stability.

- The ERM problem is underdetermined, i.e., different approximations $(\widehat{\mathbf{a}}, \widehat{b})$ may achieve similar loss for a given data set while failing to accurately model $\mathbf{a}$ and $b$. This potentially introduces error in estimating $\dot{V}$ for control inputs not reflected in the training data, and necessitates the use of exploratory control action to constrain the estimators $\widehat{\mathbf{a}}$ and $\widehat{b}$. Such exploration can be achieved by randomly perturbing the controller used in an experiment at each time step. This need for exploration is an analog to the notion of persistent 


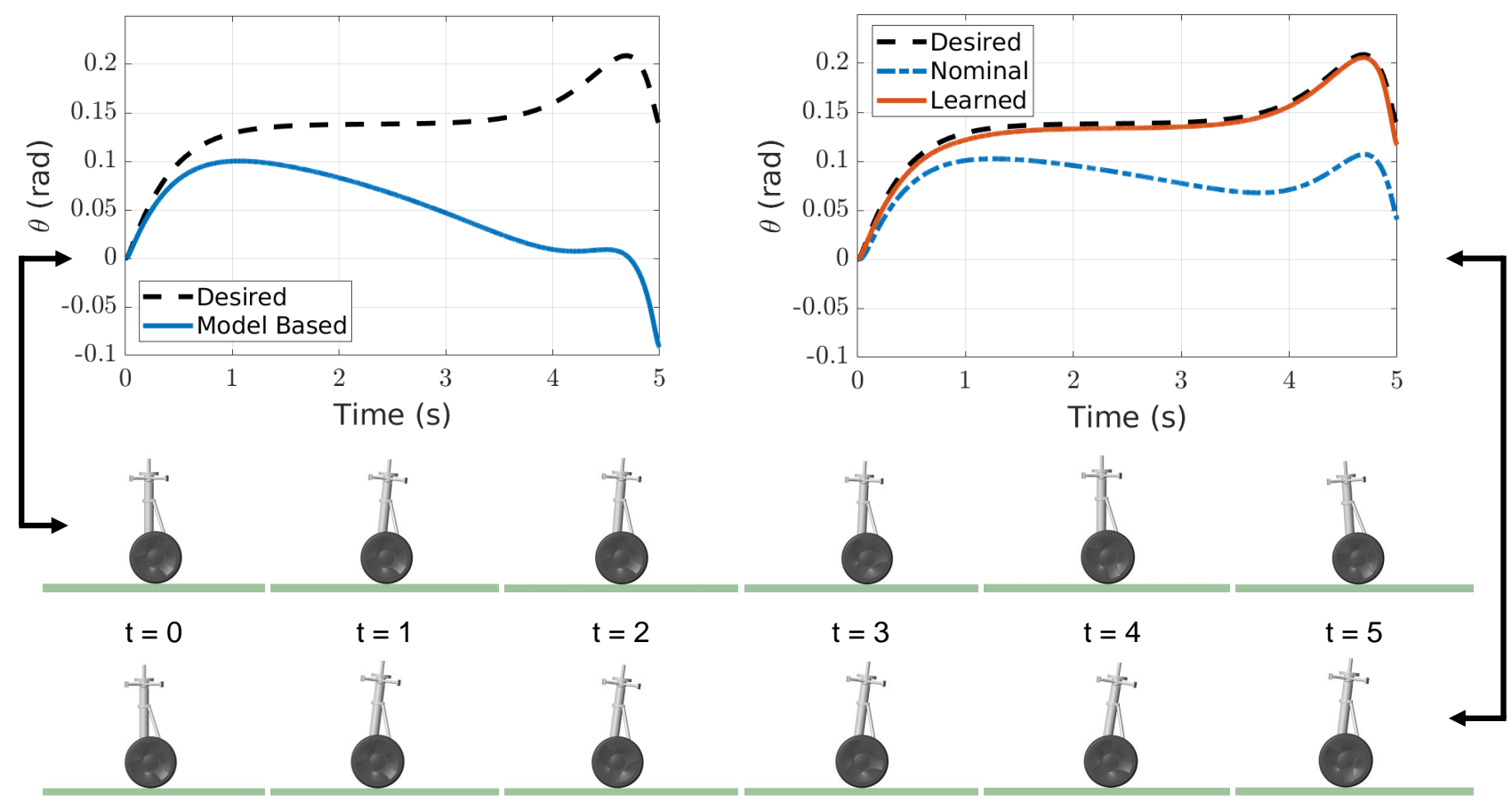

Fig. 2. (Left) Model based QP controller fails to track trajectory. (Right) Improvement in angle tracking of system with augmented controller over nominal PD controller. (Bottom) Corresponding visualizations of state data. Note that Segway is tilted in the incorrect direction at the end of the QP controller simulation, but is correctly aligned during the augmented controller simulation.

excitation from adaptive systems [28].

Algorithm 1 specifies a method of computing a sequence of Lyapunov function derivative estimates and augmenting controllers. During each episode, the augmenting controller associated with the estimate of the Lyapunov function derivative is scaled by a factor reflecting trust in the estimate and added to the nominal controller for use in the subsequent experiment. The trust coefficients form a monotonically nondecreasing sequence on the interval $[0,1]$. Importantly, this experiment need not take place in simulation; the same procedure may be executed directly on hardware. It may be infeasible to choose a specific configuration for an initial condition on a hardware platform; therefore we specify a set of initial configurations $\mathcal{Q}_{0} \subseteq \mathcal{Q}$ from which an initial condition may be sampled, potentially randomly.

\section{B. Additional Controller Details}

During augmentation, we specify the controller in 20 by selecting the minimum-norm cost function:

$$
J\left(\mathbf{u}^{\prime}\right)=\frac{1}{2}\left\|\mathbf{u}(\mathbf{q}, \dot{\mathbf{q}}, t)+\mathbf{u}^{\prime}\right\|_{2}^{2},
$$

for all $\mathbf{u}^{\prime} \in \mathbb{R}^{m}, \mathbf{q} \in \mathcal{Q}, \dot{\mathbf{q}} \in \mathbb{R}^{n}$, and $t \in \mathcal{I}$. We additionally incorporate a smoothing regularizer into the cost function of the form:

$$
R\left(\mathbf{u}^{\prime}\right)=\bar{R}\left\|\mathbf{u}^{\prime}-\mathbf{u}_{\text {prev }}\right\|_{2}^{2},
$$

for all $\mathbf{u}^{\prime} \in \mathbb{R}^{m}$, where $\mathbf{u}_{\text {prev }} \in \mathbb{R}^{m}$ is the previously computed augmenting controller and $\bar{R}>0$. This is done to avoid chatter that may arise from the optimization based nature of the CLF-QP formulation [27].

Note that for this choice of Lyapunov function, the gradient $\frac{\partial V}{\partial \boldsymbol{\eta}}$, and therefore a, approach $\mathbf{0}$ as $\boldsymbol{\eta}$ approaches 0, which occurs close to the desired trajectory. While the estimated Lyapunov function derivative may be fit with low absolute error on the data set, the relative error may still be high for states near the desired trajectory. Such relative error causes the optimization problem in 20 to be poorly conditioned near the desired trajectory. We therefore add a slack term $\delta \in \mathbb{R}_{+}$to the decision variables, which appears in the inequality constraint [3]. The slack term is additionally incorporated into the cost function as:

$$
C(\delta)=\frac{1}{2} \bar{C}\left\|\left(\frac{\partial V}{\partial \boldsymbol{\eta}} \widehat{\mathbf{g}}(\mathbf{q})\right)^{\top}+\widehat{\mathbf{a}}(\boldsymbol{\eta}, \mathbf{q})\right\|_{2}^{2} \delta^{2},
$$

for all $\delta \in \mathbb{R}_{+}$, where $\bar{C}>0$. As states approach the trajectory, the coefficient of the quadratic term decreases and enables relaxation of the exponential stability inequality constraint. In practice this leads to input-to-state stable behavior, described in [40], around the trajectory.

The exploratory control during experiments is naively chosen as additive noise from a centered uniform distribution, with each coordinate drawn i.i.d. The variance is scaled by the norm of the underlying controller to introduce exploration while maintaining a high signal-to-noise ratio. 


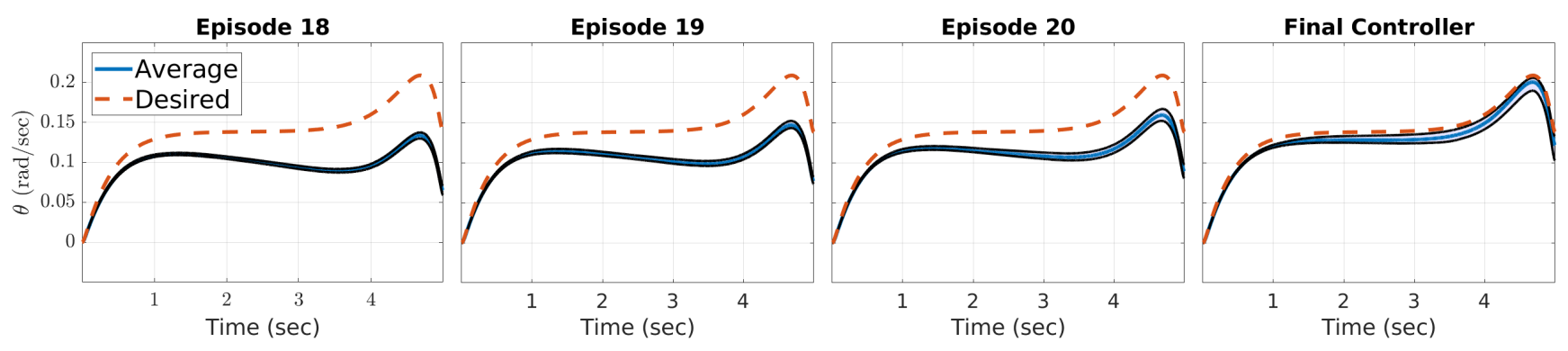

Fig. 3. Augmenting controllers consistently improve trajectory tracking across episodes. 10 instances of the algorithm are executed with the shaded region formed from minimum and maximum angles for each time step within an episode. The corresponding average angle trajectories are also displayed.

\section{APPLICATION ON SEGWAY PLATFORM}

In this section we apply the episodic learning algorithm constructed in Section IV to the Segway platform. In particular, we consider a 4-dimensional planar Segway model based on the simulation model in [18]. The system states consist of horizontal position and velocity, pitch angle, and pitch angle rate. Control is specified as a single voltage input supplied to both motors. The parameters of the model (including mass, inertias, and motor parameters but excluding gravity) are randomly modified by up to $10 \%$ of their nominal values and are fixed for the simulations.

We seek to track a pitch angle trajectory $2^{2}$ generated for the estimated model. The nominal controller is a linear proportional-derivative (PD) controller on angle and angle rate error. 20 experiments are conducted with trust values varying from 0.01 to 0.99 in a sigmoid fashion. The exploratory control is drawn uniformly at random between $-20 \%$ and $20 \%$ of the norm of the underlying controller for an episode for the first 10 episodes. The percentages decay linearly to 0 in the remaining 10 episodes. The model classes selected are sets of two-layer neural networks with ReLU nonlinearities with hidden layer width of 2000 nodes ${ }^{3}$ The inputs to both models are all states and the Lyapunov function gradient.

Failure of the controller (13) designed for the estimated model to track the desired trajectory is seen in the left portion of Fig. 2. The baseline PD controller and the augmented controller after 20 experiments can be seen in the right portion Fig. 2. Corresponding visualizations of the Segway states are displayed at the bottom of Fig. 22 The augmented controller exhibits a notable improvement over the modelbased and PD controller in tracking the trajectory.

To verify the robustness of the learning algorithm, the 20 experiment process was conducted 10 times. After each experiment the intermediate augmented controller was tested without exploratory perturbations. For the last three experiments and a test of the final augmented controller, the minimum, mean, and maximum angles across all 10 instances are displayed for each time step in Fig. 3 The mean trajectory consistently improves in these later episodes as the trust factor increases. The variation increases but

\footnotetext{
${ }^{2}$ Trajectory was generated using the GPOPS-II Optimal Control Software

${ }^{3}$ Models were implemented in Keras
}

remains small, indicating that the learning problem is robust to randomness in the initialization of the neural networks, in the network training algorithm, and in the noise added during the experiments. The performance of the controller in the earlier episodes displayed negligible variation from the baseline PD controller due to small trust factors.

LyaPy is available at https://github.com/vdorobantu/lyapy

\section{CONCLUSIONS \& FUTURE WORK}

We presented an episodic learning framework that directly integrates into an established method of nonlinear control using CLFs. Our method allows for the effects of both parametric error and unmodeled dynamics to be learned from experimental data and incorporated into an quadratic program controller. The success of this approach was demonstrated in simulation on a Segway, showing improvement upon a model estimate based controller.

There are two main interesting directions for future work. First, a more thorough investigation of episodic learning algorithms can yield superior performance as well as learningtheoretic converge guarantees. Other episodic learning approaches to consider include SEARN [13], AggreVaTeD [41], and MoBIL [11], amongst others. Second, our approach can also be applied to learning with other forms of guarantees, such as with Control Barrier Functions (CBFs) [4]. Existing work on learning CBFs are restricted to learning with Gaussian processes [43], [16], [1], and also learn over the full state space rather than over the low-dimensional projection onto the $\mathrm{CBF}$ time derivative.

\section{REFERENCES}

[1] End-to-end safe reinforcement learning through barrier functions for safety-critical continuous control tasks. 2019.

[2] Aaron D Ames, Kevin Galloway, Koushil Sreenath, and Jessy W Grizzle. Rapidly exponentially stabilizing control lyapunov functions and hybrid zero dynamics. IEEE Transactions on Automatic Control, 59(4):876-891, 2014.

[3] Aaron D Ames and Matthew Powell. Towards the unification of locomotion and manipulation through control lyapunov functions and quadratic programs. In Control of Cyber-Physical Systems, pages 219 240. Springer, 2013.

[4] Aaron D Ames, Xiangru Xu, Jessy W Grizzle, and Paulo Tabuada Control barrier function based quadratic programs for safety critical systems. IEEE Transactions on Automatic Control, 62(8):3861-3876, 2017

[5] Zvi Artstein. Stabilization with relaxed controls. Nonlinear Analysis: Theory, Methods \& Applications, 7(11):1163-1173, 1983. 
[6] Thomas Beckers, Dana Kulić, and Sandra Hirche. Stable gaussian process based tracking control of euler-lagrange systems. Automatica, 103:390-397, 2019.

[7] Felix Berkenkamp, Riccardo Moriconi, Angela P Schoellig, and Andreas Krause. Safe learning of regions of attraction for uncertain, nonlinear systems with gaussian processes. In 2016 IEEE 55th Conference on Decision and Control (CDC), pages 4661-4666. IEEE, 2016.

[8] Felix Berkenkamp and Angela P Schoellig. Safe and robust learning control with gaussian processes. In 2015 European Control Conference (ECC), pages 2496-2501. IEEE, 2015.

[9] Felix Berkenkamp, Angela P Schoellig, and Andreas Krause. Safe controller optimization for quadrotors with gaussian processes. In 2016 IEEE International Conference on Robotics and Automation (ICRA), pages 491-496. IEEE, 2016.

[10] Felix Berkenkamp, Matteo Turchetta, Angela Schoellig, and Andreas Krause. Safe model-based reinforcement learning with stability guarantees. In I. Guyon, U. V. Luxburg, S. Bengio, H. Wallach, R. Fergus, S. Vishwanathan, and R. Garnett, editors, Advances in Neural Information Processing Systems 30, pages 908-918. Curran Associates, Inc., 2017.

[11] Ching-An Cheng, Xinyan Yan, Evangelos Theodorou, and Byron Boots. Accelerating imitation learning with predictive models. In International Conference on Artificial Intelligence and Statistics (AISTATS), 2019.

[12] Yinlam Chow, Ofir Nachum, Edgar Duenez-Guzman, and Mohammad Ghavamzadeh. A lyapunov-based approach to safe reinforcement learning. In S. Bengio, H. Wallach, H. Larochelle, K. Grauman, N. Cesa-Bianchi, and R. Garnett, editors, Advances in Neural Information Processing Systems 31, pages 8092-8101. Curran Associates, Inc., 2018.

[13] Hal Daumé, John Langford, and Daniel Marcu. Search-based structured prediction. Machine learning, 75(3):297-325, 2009.

[14] Yan Duan, Xi Chen, Rein Houthooft, John Schulman, and Pieter Abbeel. Benchmarking deep reinforcement learning for continuous control. In International Conference on Machine Learning, pages 1329-1338, 2016.

[15] Damien Ernst, Pierre Geurts, and Louis Wehenkel. Tree-based batch mode reinforcement learning. Journal of Machine Learning Research, 6(Apr):503-556, 2005.

[16] Jaime F Fisac, Anayo K Akametalu, Melanie N Zeilinger, Shahab Kaynama, Jeremy Gillula, and Claire J Tomlin. A general safety framework for learning-based control in uncertain robotic systems. IEEE Transactions on Automatic Control, 2018.

[17] Kevin Galloway, Koushil Sreenath, Aaron D Ames, and Jessy W Grizzle. Torque saturation in bipedal robotic walking through control lyapunov function-based quadratic programs. IEEE Access, 3:323332, 2015.

[18] Thomas Gurriet, Andrew Singletary, Jacob Reher, Laurent Ciarletta, Eric Feron, and Aaron Ames. Towards a framework for realizable safety critical control through active set invariance. In Proceedings of the 9th ACM/IEEE International Conference on Cyber-Physical Systems, pages 98-106. IEEE Press, 2018.

[19] László Györfi, Michael Kohler, Adam Krzyzak, and Harro Walk. A distribution-free theory of nonparametric regression. Springer Science \& Business Media, 2006.

[20] Iasson Karafyllis, Maria Kontorinaki, and Miroslav Krstic. Adaptive control by regulation-triggered batch least-squares estimation of nonobservable parameters. arXiv preprint arXiv:1811.10833, 2018.

[21] H.K. Khalil. Nonlinear Systems - 3rd Edition. PH, Upper Saddle River, NJ, 2002.

[22] Jens Kober, J Andrew Bagnell, and Jan Peters. Reinforcement learning in robotics: A survey. The International Journal of Robotics Research, 32(11):1238-1274, 2013.

[23] Miroslav Krstić and Peter V Kokotović. Control lyapunov functions for adaptive nonlinear stabilization. Systems \& Control Letters, 26(1):17-23, 1995.

[24] Hoang M Le, Andrew Kang, Yisong Yue, and Peter Carr. Smooth imitation learning for online sequence prediction. In Proceedings of the 33rd International Conference on International Conference on Machine Learning-Volume 48, pages 680-688. JMLR. org, 2016.

[25] Timothy P Lillicrap, Jonathan J Hunt, Alexander Pritzel, Nicolas Heess, Tom Erez, Yuval Tassa, David Silver, and Daan Wierstra. Continuous control with deep reinforcement learning. arXiv preprint arXiv:1509.02971, 2015.

[26] Wen-Loong Ma, Shishir Kolathaya, Eric R Ambrose, Christian M Hubicki, and Aaron D Ames. Bipedal robotic running with durus2d: Bridging the gap between theory and experiment. In Proceedings of the 20th International Conference on Hybrid Systems: Computation and Control, pages 265-274. ACM, 2017.

[27] Benjamin Morris, Matthew J Powell, and Aaron D Ames. Sufficient conditions for the lipschitz continuity of qp-based multi-objective control of humanoid robots. In 52nd IEEE Conference on Decision and Control, pages 2920-2926. IEEE, 2013.

[28] Kumpati S Narendra and Anuradha M Annaswamy. Persistent excitation in adaptive systems. International Journal of Control, 45(1):127160, 1987.

[29] Quan Nguyen and Koushil Sreenath. Optimal robust control for bipedal robots through control lyapunov function based quadratic programs. In Robotics: Science and Systems, 2015.

[30] Hadi Ravanbakhsh and Sriram Sankaranarayanan. Learning lyapunov (potential) functions from counterexamples and demonstrations. arXiv preprint arXiv:1705.09619, 2017.

[31] Spencer M Richards, Felix Berkenkamp, and Andreas Krause. The lyapunov neural network: Adaptive stability certification for safe learning of dynamic systems. arXiv preprint arXiv:1808.00924, 2018.

[32] Stéphane Ross, Geoffrey Gordon, and Drew Bagnell. A reduction of imitation learning and structured prediction to no-regret online learning. In Proceedings of the fourteenth international conference on artificial intelligence and statistics, pages 627-635, 2011.

[33] Stéphane Ross, Geoffrey J. Gordon, and J. Andrew Bagnell. No-regret reductions for imitation learning and structured prediction. CoRR, abs/1011.0686, 2010.

[34] S. S. Sastry. Nonlinear Systems: Analysis, Stability and Control. Springer, NY, 1999.

[35] Stefan Schaal and Christopher G Atkeson. Learning control in robotics. IEEE Robotics \& Automation Magazine, 17(2):20-29, 2010.

[36] John Schulman, Filip Wolski, Prafulla Dhariwal, Alec Radford, and Oleg Klimov. Proximal policy optimization algorithms. arXiv preprint arXiv:1707.06347, 2017.

[37] Guanya Shi, Xichen Shi, Michael O'Connell, Rose Yu, Kamyar Azizzadenesheli, Animashree Anandkumar, Yisong Yue, and SoonJo Chung. Neural lander: Stable drone landing control using learned dynamics. In IEEE International Conference on Robotics and Automation (ICRA), 2019.

[38] E. Sontag. A 'universal' contruction of Artstein's theorem on nonlinear stabilization. Systems \& Control Letters, 13:117-123, 1989.

[39] Eduardo D Sontag. Input to state stability: Basic concepts and results. In Nonlinear and optimal control theory, pages 163-220. Springer, 2008.

[40] Eduardo D Sontag and Yuan Wang. On characterizations of the inputto-state stability property. Systems \& Control Letters, 24(5):351-359, 1995.

[41] Wen Sun, Arun Venkatraman, Geoffrey J Gordon, Byron Boots, and J Andrew Bagnell. Deeply aggrevated: Differentiable imitation learning for sequential prediction. In Proceedings of the 34th International Conference on Machine Learning-Volume 70, pages 3309-3318. JMLR. org, 2017.

[42] Jie Tan, Tingnan Zhang, Erwin Coumans, Atil Iscen, Yunfei Bai, Danijar Hafner, Steven Bohez, and Vincent Vanhoucke. Sim-toreal: Learning agile locomotion for quadruped robots. arXiv preprint arXiv:1804.10332, 2018.

[43] Li Wang, Evangelos A Theodorou, and Magnus Egerstedt. Safe learning of quadrotor dynamics using barrier certificates. In 2018 IEEE International Conference on Robotics and Automation (ICRA), pages 2460-2465. IEEE, 2018.

[44] E. R. Westervelt, J. W. Grizzle, C. Chevallereau, J. H. Choi, and B. Morris. Feedback Control of Dynamic Bipedal Robot Locomotion. Taylor \& Francis/CRC Press, 2007. 